Hautarzt 2007 · 58:68-71

DOI 10.1007/s00105-006-1160-5

Online publiziert: 13. Mai 2006

๑) Springer Medizin Verlag 2006

V. von Felbert ${ }^{1,3} \cdot$ T. Hunziker ${ }^{1} \cdot$ M. Brönnimann ${ }^{1} \cdot$ J. Roduner ${ }^{2} \cdot$ L. R. Braathen ${ }^{1}$

${ }^{1}$ Dermatologische Universitätsklinik Bern

${ }^{2}$ Niedergelassener Dermatologe, Interlaken

${ }^{3}$ Klinik für Dermatologie und Allergologie, Universitätsklinik der RWTH Aachen

\title{
Hyperpigmentierung der abdominellen Haut
}

\section{Anamnese}

Vorstellig wurde ein 19-jähriger Patient in gutem Allgemein- und Ernährungszustand mit symptomloser, großflächiger hyperpigmentierter Hautveränderung am Abdomen. Die Hautveränderung begann vor 10 Monaten und breitete sich langsam aus. Entzündungszeichen, Sonnenexposition oder der Gebrauch von Wärmflaschen bzw. Heizkissen, Medikamenteneinnahme sowie Vorerkrankungen werden verneint.

\section{Klinischer Befund}

Ungefähr $10 \times 12 \mathrm{~cm}$ großes, homogen hyperpigmentiertes Areal am Abdomen mit diskret verrukösen, papulösen Effloreszenzen mit zentraler Konfluenz und peripheren, archipelartigen, retikulären Ausläufern (• Abb. 1a).

\section{Diagnostik}

Histologie: Epidermis mit undulierender Oberfläche, Orthohyperkeratose, mäßiggradiger Akanthose mit Betonung der Epidermisabschnitte, die unterhalb der Einsenkung der Hautoberfläche liegen, leichtgradiger Hyperpigmentierung basaler Keratinozyten sowie Papillomatose ( Abb. 1b). In der papillären Dermis finden sich Teleangiektasien, eine Fragmentierung elastischer Fasern sowie ein diskretes perivaskuläres lymphozytäres Infiltrat (• Abb. 1b, c).
Direktpräparat auf Pityrosporon ovale: negativ

Labor (vom Hausarzt): normale Werte (Differenzialblutbild, Nüchternblutzucker, Kreatinin, $\gamma$-GT, Cholesterin, Kalzium, Phosphat, Albumin, TSH, Parathormon)

\section{Weiterer Verlauf}

Bei unserem Patienten trat nach 4-wöchiger Behandlung mit Minozyklin 2-mal $50 \mathrm{mg}$ täglich eine Abheilung ein, die Lokalisation des Befundes ließ sich makroskopisch nur noch erahnen. Die Minozyklin-Einnahme wurde noch weitere 2 Wochen fortgeführt. Nach über 2 Jahren wurde bisher kein Rezidiv beobachtet. 

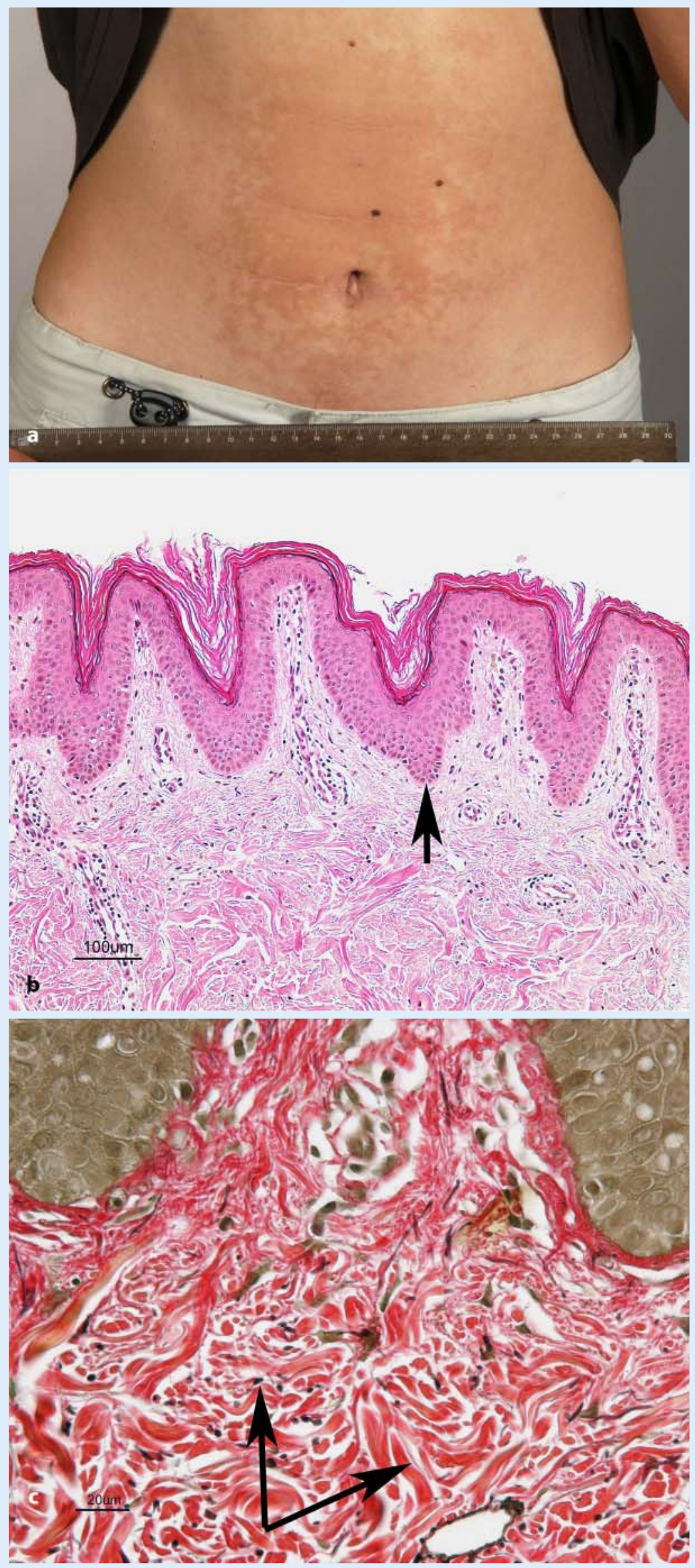

\section{Ihre Diagnose? \}

Abb. 1 a $<$ Symptomlose, in der Peripherie retikulär auslaufende Hyperpigmentierung am Abdomen. b Histologie: undulierende Epidermisoberfläche mit Orthohyperkeratose und mäßiger Akanthose v. a. unterhalb der Hautoberflächeneinsenkungen (Pfeil) sowie Teleangiektasien und lymphozytäres Infiltrat in der Dermis. HE-Färbung c Fragmentation und partieller Verlust elastischer Fasern in der Elastikavan-Gieson-Färbung (Pfeil) 


\section{จ Diagnose: Morbus Gougerot-Carteaud (Papillomatosis confluens et reticularis, PCR)}

\section{Definition}

Die Erkrankung wurde erstmals von H. Gougerot und A. Carteaud im Jahr 1927 beschrieben [2]. Es handelt sich um eine seltene Dermatose, die mit einer samtartigen bis verrukösen Hyperpigmentierung einhergeht. Sie beginnt meist intermammär und interskapulär und breitet sich flächig aus.

\section{Pathogenese}

Die Pathogenese ist nicht geklärt. Eine hereditäre Störung der Keratinisierung wird diskutiert. Dafür sprechen elektronenmikroskopische und immunhistochemische Beobachtungen [5]. Zudem wurde mehrfach ein familiäres Auftreten beschrieben [5]. Im Jahr 1969 berichteten Roberts et al. über einen möglichen Zusammenhang der Papillomatosis confluens et reticularis mit einer Pityrosporon-orbiculareBesiedelung [9]. Tatsächlich wurde bei einer Reihe von Patienten der Hefepilz Pityrosporon ovale/orbiculare nachgewiesen, und man vermutete eine mögliche Induktion der Erkrankung durch eine abnormale entzündliche Reaktion auf diesen Pilz. Dagegen spricht jedoch, dass diese Besiedelung nur bei einem Teil der Patienten nachgewiesen werden kann und dass eine topische und systemische antimykotische Behandlung meist erfolglos bleibt [7]. Hingegen führt eine systemische antibiotische Therapie mit verschiedenen Antibiotika (Tetrazykline, Fusidinsäure, Makrolide) zur Abheilung der Papillomatosis confluens et reticularis [6]. Interessanterweise berichteten kürzlich Natarajan et al. erstmals über den Nachweis einer neuen Aktinomyzeten-Spezies (Dietzia X) bei Papillomatosis confluens et reticularis [8]. Darüber hinaus wird eine Assoziation der Papillomatosis confluens et reticularis mit verschiedenen Endokrinopathien wie Diabetes mellitus, Schilddrüsenerkrankungen, Hyperpituitarismus oder Adipositas beschrieben [4]. Groh et al. vermuteten, dass die Papillomatosis confluens et reticularis eine Variante der Hautamyloi- dose sein könnte [3], während andere Autoren hinter der Papillomatosis confluens et reticularis eine Variante der Pseudoacanthosis nigricans sehen [10].

\section{Differenzialdiagnose}

Pityriasis versicolor, Pseudo-/Acanthosis nigricans, Hitzemelanose, Phytophotodermatitis.

\section{Weiterführende Diagnostik}

Für die Diagnose sind Klinik und histopathologische Befunde wegweisend. $\mathrm{Zu}$ sätzlich kann Pityrosporon ovale/orbiculare in einem Schuppenpräparat oder mittels Tesafilmabriss gesucht werden. Unterliegende Endokrinopathien sollten ausgeschlossen werden (insbesondere TSH, STH, Blutzucker, Nüchterninsulinspiegel, Androgene). Die üblicherweise anders lokalisierte Pseudoacanthosis nigricans kann bei Vorliegen einer Adipositas sowie erhöhten Nüchterninsulinwerten postuliert werden.

\section{Therapie}

Kontrollierte Therapiestudien fehlen bisher. Topische Therapien mit harnstoffund salizylsäurehaltigen Externa, Tretinoin, Antimykotika, 5-Fluorouracil, NaThiosulfat sowie Röntgenbestrahlung führten nur in Einzelfällen zum Erfolg [7]. Kasuistiken teilen eine Befundbesserung durch topisches Tazaroten, Calcipotriol oder Tacalcitol mit. Carteaud wies 1965 erstmals auf die Wirksamkeit systemischer Antibiotika hin [1]. Dies konnte von zahlreichen Autoren bestätigt werden [7]. Interessanterweise wirken verschiedene Antibiotikagruppen (Tetrazykline, Fusidinsäure, Makrolide) [6], der Wirkmechanismus ist noch unklar. Meist wird Minozyklin (2-mal täglich 50 mg für 2 Wochen bis 3 Monate) verwendet. Als Alternative gelten Makrolidantibiotika wie Azithromycin, Clarithromycin oder Erythromycin. Der erfolgreiche Einsatz von Isotretinoin (1-2 Monate) wurde ebenfalls beschrie- ben [7]. Wir haben uns bei unserem $\mathrm{Pa}$ tienten für eine systemische Therapie mit Minozyklin entschieden, da der Patient aufgrund der Großflächigkeit der Hautveränderungen in der Badesaison einen hohen Leidensdruck aufwies und die systemische antibiotische Behandlung nach Durchsicht der Literatur einer nur in Einzelfällen wirksamen Lokaltherapie deutlich überlegen ist.

\section{Verlauf}

Die Erkrankung manifestiert sich meist im jungen Erwachsenenalter. Frauen und dunkelhäutige Patienten scheinen gehäuft betroffen zu sein. Anfangs nehmen die Hyperpigmentierungen rasch $\mathrm{zu}$, bis ein stationäres Stadium erreicht wird. Spontane Besserungen wurden beschrieben [7]. Andererseits werden Wochen bis Monate nach Abheilung Rezidive beobachtet [7].

\section{Korrespondierender Autor Dr. V. von Felbert}

Klinik für Dermatologie und All-
ergologie, Universitätsklinik der
RWTH Aachen
Pauwelsstraße 30,
52074 Aachen
vvonfelbert@ukaachen.de

Interessenkonflikt. Es besteht kein Interessenkonflikt. Der korrespondierende Autor versichert, dass keine Verbindungen mit einer Firma, deren Produkt in dem Artikel genannt ist, oder einer Firma, die ein Konkurrenzprodukt vertreibt, bestehen. Die Präsentation des Themas ist unabhängig und die Darstellung der Inhalte produktneutral.

\section{Literatur}

1. Carteaud A (1965) A case of Gougerot and Carteaud's confluent and reticulated papulous papillomatosis, completely cleared up by antibiotics. Bull Soc Dermatol Syphiligr 72: 396-397

2. Gougerot H, Carteaud A (1927) Papillomatose pigmentée innominée. Bull Soc Fr Dermatol Syphiligr 34: 719-721

3. Groh V, Schnyder UW (1983) Nosologie der Papillomatose papuleuse confluente et réticulée (Gougerot-Carteaud). Hautarzt 34: 81-86 
4. Hamilton D, Tavafoghi V, Shafer JC, Hambrick GW Jr (1980) Confluent and reticulated papillomatosis of Gougerot-Carteaud: its relation to papillomatoses. J Am Acad Dermatol 2: 401-410

5. Inaloz HS, Patel GK, Knight AG (2002) Familial confluent and reticulated papillomatosis. Arch Dermatol 138: 276-277

6. Jang HS, Oh CK, Cha JH et al. (2001) Six cases of confluent and reticulated papillomatosis alleviated by various antibiotics. J Am Acad Dermatol 44: 652-655

7. Kretschmar L, Metze $\mathrm{D}$, Vehring $\mathrm{KH}$, Bonsmann $\mathrm{G}$ (1996) Papillomatosis confluens et reticularis. Hautarzt 47: 209-213

8. Natarajan S, Milne D, Jones AL et al. (2005) Dietzia strain X: a newly described Actinomycete isolated from confluent and reticulated paillomatosis. $\mathrm{Br} J$ Dermatol 153: 825-827

9. Roberts SO, Lachapelle JM (1969) Confluent and reticulate papillomatosis (Gougerot-Carteaud) and pityrosporum orbiculare. Br J Dermatol 81(11): 841-845

10. Wilhelm KP, Tronnier M, Wolff HH (1993) Pseudoacanthosis nigricans unter dem klinischen Erscheinungsbild einer Papillomatosis confluens et reticularis (Gougerot und Carteaud). Hautarzt 44: 598601

Hautarzt 2007 · 58:68-71 · DOI 10.1007/s00105-006-1268-7

Online publiziert: 13. Dezember 2006

๑) Springer Medizin Verlag 2006

\section{S. Gerdes · U. Mrowietz}

Psoriasis-Zentrum an der Klinik für Dermatologie, Venerologie und

Allergologie, Universitätsklinikum Schleswig-Holstein Campus Kiel

\section{Klassische Therapien der topischen Psoriasisbehandlung}

Hautarzt (2006) 57: 666-671

In Tab. 2 sind die Konzentrationen für die Dithranol-haltigen Fertigpräparate falsch angegeben. Die richtigen Konzentrationen sind in der folgenden Tabelle dargestellt.

Wir bitten den Fehler zu entschuldigen.

\section{Korrespondierender Autor \\ Dr. S. Gerdes}

Psoriasis-Zentrum an der Klinik für Dermatologie, Venerologie und Allergologie, Universitätsklinikum Schleswig-Holstein Campus Kiel Schittenhelmstraße 7, 24105 Kiel sgerdes@dermatology.uni-kiel.de

\section{Tab. 2 Minuten- oder Kurzkontakttherapie Dosierungsschema zugelassener Fertigprä-}

\section{parate}

\begin{tabular}{|c|c|c|c|}
\hline $\begin{array}{l}\text { Steigerungs- } \\
\text { schritte alle } 3 \\
\text { bis } 4 \text { Tage }\end{array}$ & $\begin{array}{l}\text { Psoradexan }^{\odot}(0,05 \%), \\
\text { Psoradexan }^{\odot} \text { mite }(0,1 \%) \\
\text { Psoradexan }^{\odot} \text { forte }(0,2 \%)\end{array}$ & $\begin{array}{l}\text { Psoralon }^{\odot} \text { MT 0,5, 1, } \\
2 \text { und } 3 \%\end{array}$ & Micanol $^{\odot} 1$ und $3 \%$ \\
\hline 1 & $0,05 \%$ & $1 \%$ für $10 \mathrm{~min}$ & $1 \%$ für $10 \mathrm{~min}$ \\
\hline 2 & $0,05 \%$ & $1 \%$ für $20 \mathrm{~min}$ & $1 \%$ für $20 \mathrm{~min}$ \\
\hline 3 & $0,05 \%$ & $1 \%$ für $30 \mathrm{~min}$ & $1 \%$ für $30 \mathrm{~min}$ \\
\hline 4 & $0,1 \%$ & $2 \%$ für $10 \mathrm{~min}$ & $3 \%$ für $10 \mathrm{~min}$ \\
\hline 5 & $0,1 \%$ & $2 \%$ für $20 \mathrm{~min}$ & $3 \%$ für $20 \mathrm{~min}$ \\
\hline 6 & $0,1 \%$ & $2 \%$ für $30 \mathrm{~min}$ & $3 \%$ für $30 \mathrm{~min}$ \\
\hline 7 & $0,2 \%$ & $3 \%$ für $10 \mathrm{~min}$ & $\begin{array}{l}\text { Bei zu starker Hautreizung } \\
\text { auf } 1 \% \text { zurückwechseln }\end{array}$ \\
\hline 8 & $0,2 \%$ & $3 \%$ für $20 \mathrm{~min}$ & \multirow{2}{*}{$\begin{array}{l}\text { Bei zu starker Hautreizung } \\
\text { auf } 1 \% \text { zurückwechseln }\end{array}$} \\
\hline 9 & $0,2 \%$ & $3 \%$ für $30 \mathrm{~min}$ & \\
\hline
\end{tabular}

